\title{
Impact of dietary shift to higher-antioxidant foods in COPD: a randomised trial
}

\author{
E. Keranis*, D. Makris*,\#, P. Rodopoulou*, H. Martinou*, G. Papamakarios*, \\ Z. Daniil*, E. Zintzaras ${ }^{\uparrow,+}$ and K.I. Gourgoulianis*
}

ABSTRACT: Chronic obstructive pulmonary disease (COPD) is characterised by increased oxidative stress. Dietary factors, such as ample consumption of foods rich in antioxidants, such as fruit and vegetables, might have beneficial effects in COPD patients.

The association between dietary shift to foods rich in antioxidants and lung function in COPD was investigated in a 3-yr prospective study. A total of 120 COPD patients were randomised to follow either a diet based on increased consumption of fresh fruit and vegetables (intervention group (IG)) or a free diet (control group (CG)).

The mean consumption of foods containing antioxidants was higher in the IG than in the CG throughout the study period $(p<0.05)$. The relationship between consumption of foods rich in antioxidants and percentage predicted forced expiratory volume in $1 \mathrm{~s}$ was assessed using a general linear model for repeated measures; the two groups overall were different in time $(p=0.03)$, with the IG showing a better outcome. In investigating the effect of several confounders (sex, age, smoking status, comorbid conditions and exacerbation) of group response over time, nonsignificant interactions were found between confounders, group and time.

These findings suggest that a dietary shift to higher-antioxidant food intake may be associated with improvement in lung function, and, in this respect, dietary interventions might be considered in COPD management.

KEYWORDS: Chronic obstructive pulmonary disease, diet antioxidants, epidemiology

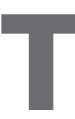
he course of chronic obstructive pulmonary disease (COPD) is characterised by progressive and irreversible airflow obstruction, impaired quality of life and increased mortality [1]. COPD prevalence is increasing worldwide, and thus management of the disease is currently considered a major health issue. Several therapeutic strategies, including smoking cessation, pharmacological interventions and rehabilitation programmes, are used with the aim of improving quality of life and decelerating lung function decline in COPD patients.

There is increasing evidence that COPD is characterised by increased airway and systemic inflammation, which is partially triggered by the aggravated oxidative stress that is prevalent in COPD patients compared to healthy subjects. Thus it has been hypothesised that balancing oxidative stress might be protective in the development of COPD and other airway diseases. Previous studies investigated the protective role of dietary interventions (based on adequate intake of antioxidants and avoidance of increasing girth) against oxidative stress [2-5]. These studies showed that foods rich in antioxidants (e.g. fruit and vegetables) may protect the lungs from oxidative damage $[6,7]$ resulting from smoking or air pollution $[8,9]$ since antioxidants are able to modulate the acute harmful effects of oxidative air pollution and smoking on the lungs [10-13]. Notably, a beneficial association between fruit intake and lung function was observed in crosssectional surveys, in which the forced expiratory volume in $1 \mathrm{~s}$ (FEV1) of subjects with increased fruit intake was $\sim 100 \mathrm{~mL}$ higher than that of subjects with decreased fruit intake $[2,14,15]$.

However, the impact of dietary supplementation on lung function has not been demonstrated in prospective longitudinal studies in COPD. In this setting, questions remain, and the role of dietary interventions based on high intake of antioxidants (i.e. a diet rich in fruit and vegetables) in COPD management remains unclear.

The primary aim of the present study was, therefore, to investigate prospectively whether a nutritional intervention consisting of a diet rich in antioxidants, such as raw fresh fruit and vegetables, would significantly affect lung function decline in COPD patients compared to a free diet.
AFFILIATIONS

Depts of *Respiratory Medicine,

${ }^{*}$ Critical Care Medicine, and

"Biomathematics, University of

Thessaly School of Medicine,

Larissa, Greece.

${ }^{+}$Center for Clinical Evidence

Synthesis, Institute for Clinical

Research and Health Policy Studies,

Tufts Medical Center, Tufts University

School of Medicine, Boston, MA,

USA

CORRESPONDENCE

D. Makris

Dept of Critical Care Medicine

University of Thessaly School

of Medicine

Biopolis

4110 Larissa

Thessaly

Greece

E-mail: appollon7@hotmail.com

Received:

July 172009

Accepted after revision:

Jan 262010

First published online:

Feb 112010 
A population of COPD patients of varying severity was randomised to receive either a diet rich in fruit and vegetables or a free diet, and the relationship between dietary intervention and lung function decline was studied over 3 yrs.

\section{METHODS}

\section{Patients}

The present investigation was a 3-yr prospective study, incorporating a recruitment period of 2 months, a run-in period and outpatient clinic visits, scheduled every 6 months. Consecutive sampling was used to recruit patients during 2004-2007, from a community-based outpatient primary medical clinic of Achillopouleio General Hospital (Volos, Greece). The clinic was based at Argalasti, a rural area in South Pelion (Greece). In order to be included in the study, patients had to have a diagnosis of COPD based on medical history, clinical signs and spirometry according to the Global Initiative for Chronic Obstructive Lung Disease (GOLD) definition, and have been free of a COPD exacerbation for $\geqslant 4$ weeks [1]. Patients with a favourable response to bronchodilators (post-bronchodilator FEV1 change of $>15 \%$ ), history of asthma, atopy, allergic rhinitis, continuous use of systemic steroids for $>30$ days in the previous year or history of lung cancer or other respiratory disease were excluded from the study. A total of 1,455 subjects who visited the outpatient clinic during the recruitment period were assessed for eligibility; 120 of those who were eligible gave their consent and entered the study (fig. 1). The study was approved by the Internal Review Board and Ethics Committee of the University of Thessaly (Larissa, Greece) and Achillopouleio General Hospital.
At baseline, all patients underwent physical examination, which included measurement of height, weight and body mass index (BMI; calculated as weight/height ${ }^{2}$ ), and were questioned regarding smoking habits, alcohol intake, dietary habits and physical activity; lung function was assessed by pre- and post-bronchodilation spirometry and comorbid conditions using the Charlson index. Respiratory symptoms, such as dyspnoea (duration and severity), sputum production, cough and wheezing, were assessed and used as additional confirmatory measurements regarding COPD diagnosis and assessment of disease severity. The baseline assessment was based on accepted diagnostic tools [16-19].

\section{Spirometry}

Every 6 months, post-bronchodilation spirometry was performed following premedication with $200 \mu \mathrm{g}$ salbutamol via a metered-dose inhaler, according to standardised guidelines [1] and using a computerised spirometer (Spirolab II; MIR, Rome, Italy). Spirometry was performed by laboratory technicians who were not aware of the study design, group allocation or study outcomes. Attention was paid to ensuring that the subject had been free of exacerbation for $\geqslant 4$ weeks on the day that the spirometry was performed.

\section{Fruit and vegetable consumption assessment}

During the present study, change in nutritional status was evaluated using a questionnaire regarding food intake and by assessing BMI. Food intake assessment was based on a previously accepted food questionnaire modified according to antioxidant food sources and local dietary habits [20-22]. It contained questions about 38 different items regarding fresh

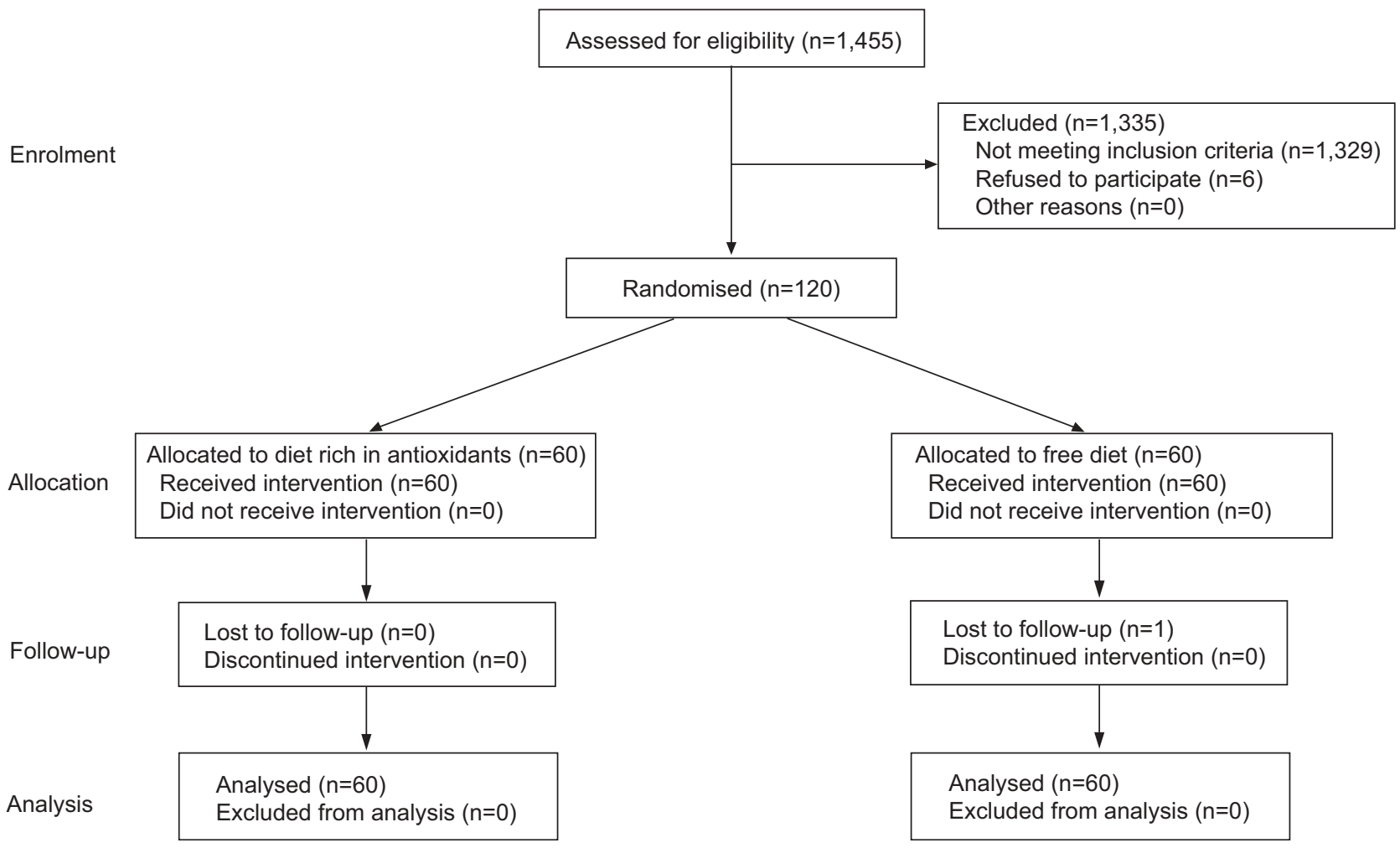

FIGURE 1. Flow diagram of the study. Nutritional enhancement in chronic obstructive pulmonary disease: a randomised controlled trial. 
salad or raw vegetables (including olives, spinach, beetroot, mushrooms, cruciferae, carrots, tomatoes, potatoes, broccoli and dark-green leafy vegetables) and fresh fruit and pure fruit juice (including strawberries, plums, apples, citrus fruits, oranges, mandarins, kiwi fruit and nuts). Most of these nutrients are produced in this rural Mediterranean region, and all of them are available all year round in this area. For each food item, patients were asked to rate their consumption on a scale of: more than once a day, once a day, most days, once or twice a week, less than once a week, or never. These responses were assigned a score ranging 5-0 accordingly, following the methods reported previously [4, 23]. An overall fresh fruit consumption score was calculated by taking the mean consumption level. This was then divided into five categories for the purposes of presentation $(0-1.00,1.01-2.00,2.01-3.00$, 3.01-4.00 and 4.01-5.00). Change in fresh fruit consumption was calculated from the absolute difference in scores. Food categories, such as overall meat, overall poultry, overall fish and overall cereal intake, were also assessed in order to identify whether the shift towards a diet rich in fruit and vegetables was associated with a decrease in other food groups.

\section{Study groups and interventions}

Patients were randomised to follow, for 3 yrs, either a diet with increased consumption of foods containing antioxidants (intervention group (IG); $n=60)$, such as fresh fruit, fruit juice and vegetables, or a free diet (control group $(C G) ; n=60$ ). Randomisation was performed using tables of random order [24] by a person (G. Papamakarios) not involved in study monitoring or outcome assessment. Two attending physicians (E. Keranis and E. Meli) and two specialist nurses (H. Martinou and G. Kotopouli) were responsible for seeing and monitoring the patients in both groups and were blind to outcome assessment. It was scheduled for both groups to be seen equally by all physicians/nurses. IG patients were seen regularly in the outpatient clinic (scheduled outpatient visits every 6 months), where they were informed of the potential beneficial effects of fruit and vegetables on their health status by two members of the study team (one of the two attending physicians and one of the two specialist nurses). At baseline and at each visit, it was clearly explained to them that the dietary goal was to increase fresh fruit/fruit juice/vegetable consumption by $\geqslant 1$ portion. day $^{-1}$ compared to baseline based on the results of the MORGEN study (Monitoring project on risk factors and health in the Netherlands) and local dietary habits and to maintain this regimen throughout the study period $[15,23]$. Patients in the CG were also seen regularly (scheduled outpatient visits every 6 months) in the outpatient clinic by two members of the study team (one of the two attending physicians/nurses) and received the standard care for COPD, but dietary issues were not discussed. The dietary questionnaire was repeated at each outpatient visit. All patients were permitted to contact the study team each time they experienced deterioration in their clinical condition. COPD exacerbations were treated according to published guidelines [1] and recorded at each visit. Outcome was assessed by senior members of the research team (K.I. Gourgoulianis and D. Makris).

\section{Statistical analysis}

On analysis, the dependant variable was change in postbronchodilation FEV1 expressed as a percentage of the predicted value, as previously described [19]. The two groups (CG and IG) were compared using a general linear model for repeated measures with Bonferroni adjustment. The model uses the FEV1 as response, and includes the following sources: group effect, patients within-group effect, follow-up (time) effect, interaction between follow-up and group, and error. The effect of possible confounders (sex, age (cut-off 65 yrs), smoking status (ever/never), morbid conditions (using Charlson index values) and exacerbations (frequent exacerbators or not according to median value [19])) was also assessed by including each confounding effect (in turn) and its interaction with group and time in the above model. The power for detecting the overall observed mean difference in FEV1 between the two groups based on the sample estimated $\mathrm{SD}$ at a significance level of $5 \%$, given that 60 patients were allocated to each group, was calculated. The association between excess of fruit/vegetable consumption and improvement in FEV1 was tested using Fisher's exact test. The normality of the response variable FEV1 at each time-point was tested using the Kolmogorov-Smirnov test. A result was considered significant when $\mathrm{p}<0.05$. Data were analysed using the SPSS v12 (SPSS, Inc., Chicago, IL, USA).

\section{RESULTS}

The baseline characteristics of participants are presented in table 1 . The participants' mean \pm SEM age was $68.1 \pm 1.4 \mathrm{yrs}$, and $105(87.5 \%)$ out of 120 were male. According to GOLD COPD severity, $22(18.5 \%)$ patients were stage I, $79(65.8 \%)$ stage II, $15(12.5 \%)$ stage III and four (3.3\%) stage IV. Table 1 demonstrates that there were no significant differences between the two groups (IG and CG) at baseline with respect to demographic, smoking and dietary habits, clinical characteristics and spirometric results. The participants attended 794 (394 IG and 400 CG) out of 840 6-monthly scheduled visits. There was no difference between the IG and CG in respect of the frequency with which they were seen by each attending physician (Chi-squared test; $\mathrm{p}=0.38)$. A total of $23(19.1 \%)$ patients stopped smoking during the study. There was no significant difference between the proportion of patients who quit smoking in the IG and CG. There were no significant changes in BMI and exercise between the two groups. There was also no significant difference regarding alcohol consumption between the two groups throughout the study. The mean \pm SEM annual exacerbation rates in the year preceding the study were $0.45 \pm 0.1$ and $0.51 \pm 0.1$ in the IG and CG, respectively $(\mathrm{p}=0.1)$, and the mean annual rates during the study were $0.18 \pm 0.09$ and $0.34 \pm 0.07$, respectively $(p=0.001)$. Three deaths occurred during the last 6 months of the study, all in the IG $(p=0.24)$.

\section{Fresh fruit and vegetable consumption}

Figures 2 and 3 show that patients in the IG increased their mean annual consumption of fresh fruit and vegetables significantly compared to the CG. There was no significant difference in compliance regarding the suggested and measured intake of fresh fruit and vegetables (Mann-Whitney Utest; $\mathrm{p}=0.72$ ). In the IG, the meat consumption score was reduced at the end of the study compared to baseline $(1.90 \pm 0.3$ versus $1.71 \pm 0.3$, respectively; $p=0.45$; Wilcoxon signed-rank test), whereas, in the $C G$, no significant difference was found ( $1.87 \pm 0.4$ versus $1.91 \pm 0.3 ; p=0.7)$. 


\begin{tabular}{|c|c|c|c|c|}
\hline \multirow[t]{2}{*}{ TABLE 1} & \multirow[b]{2}{*}{ Overall } & \multirow[b]{2}{*}{ IG } & \multirow[b]{2}{*}{ CG } & \multirow[b]{2}{*}{ p-value } \\
\hline & & & & \\
\hline Subjects n & 120 & 60 & 60 & \\
\hline Males/females n & $105 / 15$ & $54 / 6$ & $51 / 9$ & 0.58 \\
\hline Age yrs & $68.1 \pm 1.40$ & $66.1 \pm 1.46$ & $70.2 \pm 1.15$ & 0.07 \\
\hline $\mathrm{BMI} \mathbf{k g} \cdot \mathrm{m}^{-2}$ & $28.60 \pm 0.41$ & $28.20 \pm 0.60$ & $29.02 \pm 0.66$ & 0.35 \\
\hline Current smoker & 37 (30.8) & 17 (28.3) & 20 (33.3) & 0.69 \\
\hline Ex-smoker & $76(63.3)$ & $39(65.0)$ & $37(61.6)$ & 0.84 \\
\hline Smoking pack-yrs & $68 \pm 3.79$ & $62.4 \pm 5.03$ & $73.6 \pm 5.62$ & 0.14 \\
\hline \multicolumn{5}{|l|}{ Consumption score } \\
\hline Vegetable & $1.22 \pm 0.3$ & $1.26 \pm 0.4$ & $1.18 \pm 0.4$ & 0.30 \\
\hline Fresh fruit/juice & $1.42 \pm 0.4$ & $1.22 \pm 0.4$ & $1.48 \pm 0.5$ & 0.70 \\
\hline Meat & $1.87 \pm 0.2$ & $1.90 \pm 0.3$ & $1.87 \pm 0.4$ & 0.70 \\
\hline Fish & $1.05 \pm 0.2$ & $1.11 \pm 0.3$ & $1.02 \pm 0.4$ & 0.60 \\
\hline Alcohol & $1.61 \pm 0.3$ & $1.30 \pm 0.3$ & $1.18 \pm 0.3$ & 0.17 \\
\hline Chronic cough & $89(74)$ & $45(75)$ & $44(72)$ & 0.61 \\
\hline Chronic sputum & $86(71)$ & $39(65)$ & $47(78)$ & 0.39 \\
\hline Chronic dyspnoea & $52(56)$ & $28(46)$ & $24(40)$ & 0.75 \\
\hline FEV 1 L & $1.82 \pm 0.06$ & $1.93 \pm 0.10$ & $1.71 \pm 0.07$ & 0.09 \\
\hline FEV $1 \%$ pred & $64.28 \pm 1.58$ & $64.39 \pm 2.32$ & $64.17 \pm 2.19$ & 0.94 \\
\hline FVC L & $2.75 \pm 0.09$ & $2.90 \pm 0.14$ & $2.59 \pm 0.11$ & 0.09 \\
\hline FVC $\%$ pred & $74.64 \pm 1.66$ & $75.71 \pm 2.47$ & $73.50 \pm 2.22$ & 0.52 \\
\hline FEV $1 / F V C \%$ pred & $64.67 \pm 0.96$ & $64.77 \pm 1.14$ & $64.57 \pm 1.56$ & 0.92 \\
\hline PEF L. $\min ^{-1}$ & $4.62 \pm 0.17$ & $4.98 \pm 0.28$ & $4.26 \pm 0.21$ & 0.10 \\
\hline $\mathrm{Sa}, \mathrm{O}_{2} \%$ & $95.58 \pm 0.24$ & $95.20 \pm 0.33$ & $95.96 \pm 0.34$ & 0.81 \\
\hline Charlson index & $2.58 \pm 0.12$ & $2.39 \pm 0.16$ & $2.77 \pm 0.17$ & 0.11 \\
\hline Arthritis & $1(0.8)$ & $0(0.0)$ & $1(1.7)$ & 1 \\
\hline Cardiac failure & $25(20.8)$ & $10(16.7)$ & $15(25.0)$ & 0.36 \\
\hline Coronary disease & $33(27.5)$ & $16(26.7)$ & $17(28.3)$ & 1 \\
\hline Diabetes & $22(18.3)$ & $8(13.3)$ & $14(23.3)$ & 0.23 \\
\hline Diseases of the liver & $2(1.7)$ & $0(0.0)$ & $2(3.3)$ & 0.49 \\
\hline Hypertension & $69(57.5)$ & $32(53.3)$ & $37(61.7)$ & 0.46 \\
\hline Nephropathy & $3(2.7)$ & $2(1.7)$ & $1(3.3)$ & 1 \\
\hline Stroke & $8(6.7)$ & $4(6.7)$ & $4(6.7)$ & 1 \\
\hline
\end{tabular}

Data are presented as mean \pm SEM or $n(\%)$, unless otherwise indicated. The Mann-Whitney test or Fisher's exact test was used as appropriate for comparisons between groups. IG: intervention group; CG: control group; BMI: body mass index; FEV1: forced expiratory volume in $1 \mathrm{~s}$; \% pred: \% predicted; FVC: forced vital capacity; PEF: peak expiratory flow; $\mathrm{Sa}, \mathrm{O}_{2}$ : arterial oxygen saturation.

The response variable FEV1 was normally distributed at each time point $(p>0.20)$. The mean annual rate of FEV1 change in the entire population was $0.35 \%$ pred $(95 \%$ confidence interval $-0.22-0.55 \%$ pred), corresponding to an absolute value of 63 $(-41-93) \mathrm{mL} \cdot \mathrm{yr}^{-1}$. Spirometric values in IG patients (FEV1, FVC and FEV1/FVC) were found to be significantly higher at the end of the study. The association between frequency of consumption of various fruit and vegetables rich in antioxidants and mean percentage predicted FEV1 change per 6 months are shown in table 2. Excess antioxidant consumption and improvement in FEV1 was significantly associated $(p<0.01)$.

The general linear model for repeated measures analysis revealed that the two groups (IG and CG) overall were

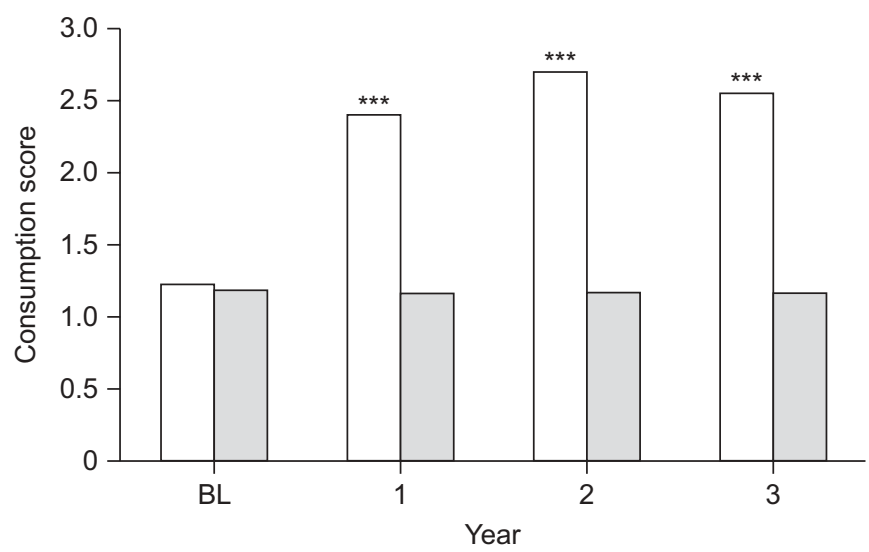

FIGURE 2. Annual vegetable consumption score in patients who followed a diet rich in fruit and vegetables $(\square)$ and in those who followed a free diet during the study $(\square)$. Data are presented as means; differences at baseline (BL) were nonsignificant. ***: $\mathrm{p}<0.001$ versus control.

different with time $(\mathrm{p}=0.03)$, with the IG showing a better outcome (fig. 4). However, the differences between the two groups were not consistent with time, i.e. there was an interaction between group effect and time, and the difference in FEV1 between groups tended to increase over time $(p<0.01)$. In investigating the effect of confounders (sex, age, smoking status, comorbid conditions and exacerbations) in group response over time, nonsignificant interactions were found between the confounders, group and time $(p=0.81, p=0.16$, $\mathrm{p}=0.77, \mathrm{p}=0.48$ and $\mathrm{p}=0.43$, respectively).

\section{DISCUSSION}

In the present prospective randomised study, the association between lung function decline and dietary shift to foods rich in antioxidants was evaluated in a population consisting of 120 patients with COPD, for a total follow-up period of $3 \mathrm{yrs}$. The main finding of the present study was that COPD patients who followed a diet with a high intake of fruit and vegetables showed an annual increase in percentage predicted FEV1, whereas patients who were on a standard diet exhibited a

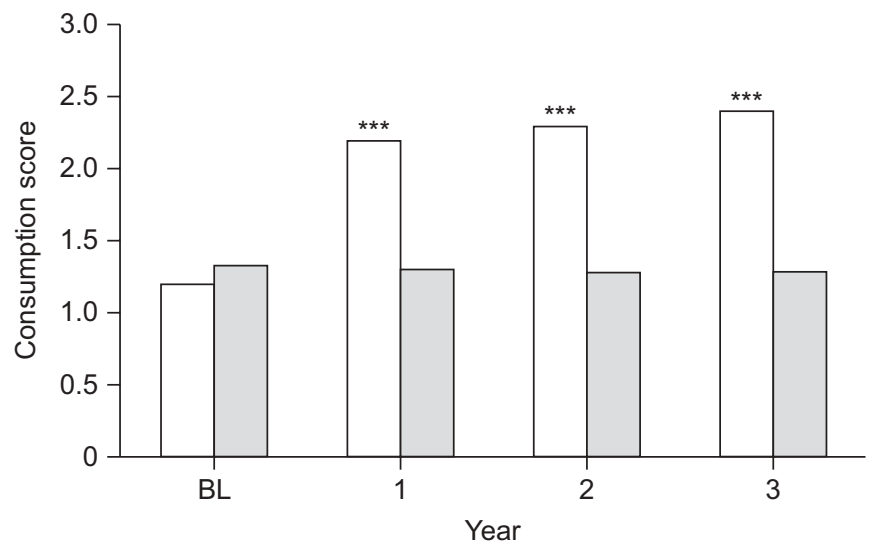

FIGURE 3. Annual fresh fruit consumption score in patients who followed a diet rich in fruit and vegetables $(\square)$ and in those who followed a free diet during the study ( $\square$ ). Data are presented as means; differences at baseline (BL) were nonsignificant. ${ }^{* *}: \mathrm{p}<0.001$ versus control. 


\begin{tabular}{llll} 
TABLE 2 & $\begin{array}{l}\text { Association between frequency of consumption } \\
\text { of fruit and vegetables rich in antioxidants and } \\
\text { forced expiratory volume in } 1 \text { s change } \\
\text { chronic obstructive pulmonary disease }\end{array}$ \\
& More than twice a week & Twice a week or less & p-value \\
\hline & $1.14(0.93-1.36)$ & $-0.54(-1.02--0.43)$ & 0.0001 \\
Olives (black) & $1.15(0.95-1.35)$ & $-0.85(-1.10--0.60)$ & 0.0001 \\
Spinach & $1.03(0.68-1.23)$ & $-0.93(-1.15--0.65)$ & 0.12 \\
Mushrooms & $1.09(0.86-1.32)$ & $-0.90(-1.15--0.66)$ & 0.07 \\
Beet & $1.14(0.89-1.13)$ & $-0.55(-0.76--0.21)$ & 0.0005 \\
Broccoli & $1.11(0.91-1.30)$ & $-0.91(-1.17--0.66)$ & 0.0001 \\
Radicchio & $1.19(0.97-1.14)$ & $-0.64(-0.9--0.37)$ & 0.005 \\
Strawberries & $1.00(0.76-1.23)$ & $-1.01(-1.12--0.77)$ & 0.0001 \\
Oranges & $1.09(0.88-1.29)$ & $-0.89(-1.11--0.64)$ & 0.0001 \\
Plums & $0.57(-0.29-0.84)$ & $-0.90(-1.23--0.57)$ & 0.0003 \\
Grapes (yel- & & & \\
\multicolumn{1}{l}{ low) } & $0.35(-0.38-1.32)$ & $-0.41(-1.12-0.32)$ & 0.1 \\
\hline Tangerines & & & \\
\hline
\end{tabular}

Data are presented as mean ( $95 \%$ confidence interval). ${ }^{*}$ : change in mean percentage predicted value per 6 months; $^{\circ}$ : Mann-Whitney U-test.

decrease in FEV1 over 3 yrs (general linear model for repeated measures; $p=0.03$ ). The present results are consistent with the results of previous studies that have investigated the effect of antioxidant intake on lung function [2, 6, 15, 25], despite the differences in design and study population between the present and former studies.

In the present investigation, it was found that a dietary shift to nutrition richer in antioxidants foods may have a significant impact upon lung function. Patients who changed their diet from low to modest consumption of fruit and vegetables (IG) exhibited an increase in FEV1, which is a parameter of lung function that characterises the course of COPD. In contrast,

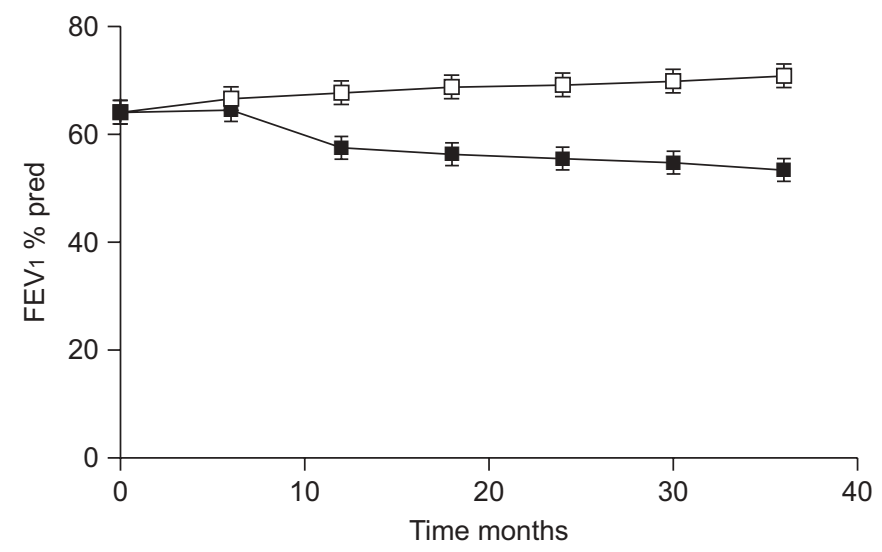

FIGURE 4. Change in forced expiratory volume in $1 \mathrm{~s}$ (FEV1) over the 3-yr dietetic intervention study period in patients who followed a diet rich in fruit and vegetables $(\square)$ and in those who followed a free diet $(\boldsymbol{\square})$. Data are presented as mean \pm SEM. The difference in mean annual decline in FEV 1 between the two groups obtained by a general linear model for repeated measures with Bonferroni adjustment gave a p-value of 0.03 . \% pred: \% predicted. patients in the CG exhibited a decline in lung function. The impact of dietary shift to higher-antioxidant food intake may have positively affected lung function in the present COPD patients by balancing oxidative stress and reducing lung inflammation.

Fruit and vegetables contain significant amounts of antioxidant vitamins, such as vitamins $C$ and $E$ and $\beta$-carotene, which may protect the lungs from oxidative damage due to smoking and air pollution. There is evidence from previous studies that these vitamins exhibit antioxidant and anti-inflammatory properties [26]. Vitamin C is present in intracellular and extracellular lung fluids and supports the normal hydration of airway surfaces. Its deficiency may lead to dry sticky mucous membranes lining the airway, and thus low levels of vitamin C may play a role in the progression of inflammation in airway diseases. Vitamin $\mathrm{E}$ is present in extracellular lung fluid and lipid membranes, where it converts oxygen radicals and lipid peroxyl radicals to less-reactive forms. $\beta$-Carotene is a free radical scavenger present in tissue membranes.

In this respect, previous studies have investigated whether the consumption of antioxidants affects lung function [7, 9, 12, 14]. However, these studies had an observational design and used populations with respiratory symptoms, but not specifically COPD patients. In addition, they used end-points such as bronchial reactivity, which are applicable in asthma rather than in COPD. Thus it was difficult to draw firm conclusions. In the present prospective randomised study, COPD patients from among 1,455 subjects who visited the outpatient clinic during the recruitment period were included, and, according to a recent Greek study, this population might represent the general COPD population in this region [27]. Thus the present findings, which showed a relationship between the consumption of food rich in antioxidants and lung function in COPD patients, might be considered in the management of COPD.

It might be argued that lung function in the IG might have been positively affected by other factors not related to dietary shift to higher-antioxidant foods, such as fruit and vegetables. The possibility certainly cannot be excluded that other dietary, environmental and genetic factors or the presence of comorbid conditions may have affected the course of lung function in the IG patients. For example, the addition of fruit and vegetables in the regimen of IG patients resulted in decreased consumption of meat, which is known to increase the burden of oxidative stress $[26,28]$. In addition, persons who initiate a preventive therapy (such as a dietary regimen based on fruit and vegetables, which might be considered as healthy) may also adhere to other social health behaviours that may also decrease the risk of adverse outcomes. Almost $47 \%$ of the patients in the present study had cardiovascular problems, and a diet rich in fruit and vegetables might have been helpful to the status of these patients by improving muscle mass and performance $[29,30]$. However, in the present study, several factors that could have acted as confounding variable biases were assessed, such as changes in physical activity, alcohol intake, comorbid conditions and exacerbation frequency, and no significant difference was found between the two groups.

Furthermore, the population studied represented mainly COPD patients followed at the same health centre, and both 
population groups were, in many respects, homogenous (white Mediterranean inhabitants of a rural peninsula with similar baseline spirometric indices and clinical status). Thus, it was assumed that all of the subjects were affected by the same environmental factors and that comorbid conditions have not affected the present results.

Another alternative explanation for the relationship that was found between a dietary shift to higher-antioxidant foods and lung function in the present study could be that patients who were marginally malnourished recovered with additional energy input and, therefore, their respiratory performance improved. However, baseline dietary status was similar in both groups in the present study (figs 2 and 3), and neither changes in BMI, smoking status nor social activities support this alternative explanation.

We certainly acknowledge that the beneficial impact of fruit and vegetable intake on lung function might be due to another bioactive nutrient and not solely due to the presence of antioxidants in fruit and vegetables. A limitation of the present study is that oxidative stress was not assessed using blood, urine or exhaled breath condensate markers, which would provide further insight into our hypothesis. This might certainly be a point of interest that could be adequately assessed in the future.

A total of $19 \%$ of patients stopped smoking during the present study. KANNER et al. [30] analysed the Lung Health Study data and reported that patients who permanently quit smoking showed a deceleration in lung function decline, or even an improvement in lung function, within years. However, it is unlikely that smoking cessation would have affected the present results. There was no significant difference between the proportion of patients who quit smoking in the IG and CG in the present study and, therefore, the effect of smoking on lung function may have affected both groups similarly.

Patients in the IG group presented with fewer exacerbations compared to the CG during the present study. In this respect, it might be argued that exacerbation frequency might have a significant impact upon lung function changes in the two groups. However, in investigating the effect of confounders of group response over time, no significant interactions were found between exacerbations, patient group and time. Nevertheless, it should be emphasised that exacerbations were assessed by symptom questioning alone in the present study and exacerbation rates were lower than in previous studies in which more rigorous methodology based on diary card data was used [19]. Therefore, patients might have underreported exacerbations in the present study, and this limitation should be considered in the interpretation of the results.

In this prospective longitudinal investigation, data were collected by interviewing patients and using questionnaires, and, accordingly, compliance with the suggested dietary regimen was assessed. We certainly acknowledge that, although the intake of various fruit and vegetables was assessed, other food groups, such as meat, poultry, fish and cereal, were assessed only as a group (e.g. overall meat intake). This fact did not permit evaluation of further parameters, such as total energy intake, which could provide further information regarding the present population. However, the primary aim was to augment the consumption of foods rich in antioxidants, such as fruit and vegetables, which are readily available and less costly than meat or fish in this particular geographical area.

It might be argued that using frequency questionnaires to assess consumption of food might not be as precise as $24-\mathrm{h}$ recall or 7 day recall, which may permit better assessment of the adopted diet. In addition, apart from the questionnaire used, another formal evaluation (i.e. home visits or 24-h diary cards), which could provide further verification regarding compliance with the advised diet, was not undertaken. Nevertheless, frequency questionnaires are an accepted methodology that have been widely applied in previous longitudinal COPD studies [30] and easier to apply in the present setting than the 24-h recall method.

A final point that should also be noted is that the present study is an open-label study. Despite the persons who gave the advice regarding fruit and vegetable consumption not being involved in outcome assessment, they were aware of group allocation and this fact has to be considered in the interpretation of the findings.

In conclusion, in the present prospective randomised trial, it was demonstrated, by using long-term follow-up and adequate control subjects, that increased intake of fruit and vegetables may have a protective effect on FEV1 in patients with COPD. We certainly acknowledge that the effects of a high fruit and vegetable intake on FEV1 might be too small to result in changes in clinically manifest disease. Future investigations should assess whether or not a dietary shift to higherantioxidant foods also has an impact on outcome measures, other than lung function, which are important to patients' perception of their disease, such as quality of life, function or perceived dyspnoea. Nevertheless, the present findings suggest that a diet rich in antioxidants, such as fruit and vegetables, may be associated with favourable outcomes in COPD, and, in this respect, the place of dietary interventions in the management of COPD warrants consideration.

\section{SUPPORT STATEMENT}

This study was supported by the University of Thessaly, Larissa, Greece.

\section{CLINICAL TRIAL}

This study is registered at ClinicalTrials.gov (trial number NCT00884299).

\section{STATEMENT OF INTEREST}

None declared.

\section{ACKNOWLEDGEMENTS}

The work was performed at Argalasti Health Centre (Achillopouleio General Hospital, Volos, Greece) under the supervision of the Dept of Respiratory Medicine (University of Thessaly School of Medicine, Larissa, Greece).

The authors wish to thank N. Tzanakis (University of Crete, Heraklion, Greece) for valuable contribution to the design of the study, and G. Kotopouli and E. Meli and all the medical and nursing personnel of Argalasti Health Centre (Achillopouleio General Hospital, Volos, Greece) for their participation in this study. 


\section{REFERENCES}

1 Pauwels RA, Buist AS, Peter M, et al. Global strategy for the diagnosis, management and prevention of chronic obstructive pulmonary disease. NHLBI/WHO Global Initiative for Chronic Obstructive Lung Disease (GOLD) workshop summary. Am J Respir Crit Care Med 2001; 163: 1256-1276.

2 Tabak C, Smit HA, Rasanen L, et al. Dietary factors and pulmonary function: a cross sectional study in middle aged men from three European countries. Thorax 1999; 54: 1021-1026.

3 Britton JR, Pavord ID, Richards KA, et al. Dietary antioxidant vitamin intake and lung function in the general population. Am J Respir Crit Care Med 1995; 151: 1383-1387.

4 Ness AR, Khaw KT, Bingham S, et al. Vitamin C status and respiratory function. Eur J Clin Nutr 1996; 50: 573-579.

5 Dow L, Tracey M, Villar A, et al. Does dietary intake of vitamins C and $\mathrm{E}$ influence lung function in old people? Am J Respir Crit Care Med 1996; 154: 1401-1404.

6 Grievink L, Smit HA, Ocke MC, et al. Dietary intake of antioxidant (pro)-vitamins, respiratory symptoms and pulmonary function: the MORGEN study. Thorax 1998; 53: 166-171.

7 Schwartz J, Weiss ST. Dietary factors and their relation to respiratory symptoms. The Second National Health and Nutrition Examination Survey. Am J Epidemiol 1990; 132: 67-76.

8 Heffner JE, Repine JE. Pulmonary strategies of antioxidant defense. Am Rev Respir Dis 1989; 140: 531-554.

9 Chatham MD, Eppler JH Jr, Saunder LR, et al. Evaluation of the effects of vitamin $\mathrm{C}$ on ozone induced bronchoconstriction in normal subjects. Ann N Y Acad Sci 1987; 498: 269-279.

10 Mohsenin V. Effect of vitamin $\mathrm{C}$ on $\mathrm{NO}_{2}$-induced airway hyperresponsiveness in normal subjects. Am Rev Respir Dis 1987; 136: 1408-1411.

11 Mohsenin V. Lipid peroxidation and antielastase activity in the lung under oxidant stress: role of antioxidant defenses. J Appl Physiol 1991; 70: 1456-1462.

12 Bucca C, Rolla G, Farina JC. Effect of vitamin C on transient increase of bronchial responsiveness in conditions affecting the airways. Ann N Y Acad Sci 1992; 669: 175-187.

13 Romieu I, Meneses F, Ramirez M, et al. Antioxidant supplementation and respiratory functions among workers exposed to high levels of ozone. Am J Respir Crit Care Med 1998; 158: 226-232.

14 Strachan DP, Cox BD, Erzinclioglu SW, et al. Ventilatory function and winter fresh fruit consumption in a random sample of British adults. Thorax 1991; 46: 624-629.

15 Tabak C, Smit HA, Heederik D, et al. Diet and chronic obstructive pulmonary disease: independent beneficial effects of fruits, whole grains, and alcohol (the MORGEN study). Clin Exp Allergy 2001; 31: 747-755.

16 Martinez JA, Straccia L, Sobrani E, et al. Dyspnea scales in the assessment of illiterate patients with chronic obstructive pulmonary disease. Am J Med Sci 2000; 320: 240-243.

17 Ferris BG. Epidemiology Standardization Project. II. Recommended respiratory disease questionnaires for use with adults and children in epidemiological research. Am Rev Respir Dis 1978; 118: 7-57.

18 Charlson M, Szatrowski T, Peterson J, et al. Validation of a combined comorbidity index. J Clin Epidemiol 1994; 47: 1245-1251.

19 Makris D, Moschandreas J, Damianaki A, et al. Exacerbations and lung function decline in COPD: New insights in current and exsmokers. Respir Med 2007; 101: 1305-1312.

20 Block G, Hartman AM, Dresser CM, et al. A data-based approach to diet questionnaire design and testing. Am J Epidemiol 1986; 124 453-469.

21 Pellegrini N, Serafini M, Colombi B, et al. Total antioxidant capacity of plant foods, beverages and oils consumed in Italy assessed by three different in vitro assays. J Nutr 2003; 133: 2812-2819.

22 Chatzi L, Apostolaki G, Bibakis I, et al. Protective effect of fruits, vegetables and the Mediterranean diet on asthma and allergies among children in Crete. Thorax 2007; 62: 677-683.

23 Brug J, Schols A, Mesters I. Dietary change, nutrition education and chronic obstructive pulmonary disease. Patient Educ Couns 2004; 52, 3: 249-257.

24 Mead R, Curnow RN. Statistical Methods in Agriculture and Experimental Biology. Texts in Statistical Science. London, Chapman and Hall/CRC, 1983; p. 34.

25 Watson L, Margetts B, Howarth $\mathrm{P}$, et al. The association between diet and chronic obstructive pulmonary disease in subjects selected from general practice. Eur Respir J 2002; 20: 313-318.

26 Lippman RD. Free radical-induced lipoperoxidation and aging. In Miquel J, Quintanilha AT, Weber H, eds. Handbook of Free Radicals and Antioxidants in Biomedicine. Boca Raton, CRC Press, 1989; pp. 187-197.

27 Tzanakis N, Anagnostopoulou U, Filaditaki V, et al. Prevalence of COPD in Greece. Chest 2004; 125: 892-900.

28 Balabanidis A. Free radicals and their role in biological systems. Beta Med Arts 2006; 8: 160-162.

29 Wouters EF. Eat well to get well. Thorax 2003; 58: 739-740.

30 Kanner RE, Anthonisen NR, Connet JE. Lower respiratory illnesses promote FEV1 decline in current smokers but not exsmokers with mild chronic obstructive pulmonary disease. Results from the Lung Health Study. Am J Respir Crit Care Med 2001; 164: 358-364. 